\title{
The Higgs Physics Programme at the International Linear Collider
}

\author{
Claude Dürig ${ }^{* \dagger}$ \\ DESY \\ E-mail: claude.duerig@desy.de
}

In the Standard Model (SM), the couplings of the Higgs boson are specified once its mass is known. One of the key ingredients of the physics programme of the International Linear Collider (ILC) are direct and model-independent precision measurements of the Higgs properties, which can either verify the mechanism of electroweak symmetry breaking in the SM or uncover new physics phenomena. In this article we give an overview of the Higgs physics programme at the ILC in view of the recently published running scenarios. In particular, we focus on fully modelindependent measurements of Higgs gauge boson couplings, the total Higgs width, as well as the top-Yukawa and the Higgs self-coupling.

The European Physical Society Conference on High Energy Physics

22-29 July 2015

Vienna, Austria

* Speaker.

${ }^{\dagger}$ On behalf of the ILC physics and detector study. 


\section{Introduction}

The discovery of a Higgs-like boson at the LHC $[1,2]$ in 2012 was a milestone in the history of particle physics. Now, after the discovery, it is crucial to investigate its nature and its role in the SM. Therefore, we require a model-independent reconstruction of the electroweak symmetry breaking sector and the verification of theoretical SM predictions, any deviation of which would be an indication of new physics beyond the SM. Depending on the new physics model we expect deviations from the SM Higgs couplings up to the few-\% level. Consequently, it is essential to reconstruct the electroweak symmetry breaking sector completely model-independent with high precision measurements. In this article we give an overview of the ILC Higgs physics programme in view of the recently updated running scenario [3]. A detailed description of the full Higgs physics programme can be found $[4,5]$.

The ILC represents one of the largest next generation collider projects. The collider is planned to be operating at a tuneable energy between $250 \mathrm{GeV}$ and $500 \mathrm{GeV}$, upgradeable to $1 \mathrm{TeV}$. Running with various beam polarisations, each contributing important aspects to the different Higgs measurements, is one of the main features of the ILC. A Technical Design Report (TDR) [5, 6, 7, 8, 9] was published in 2012. Japan indicates great interest to host the ILC. Site specific investigations selected the Kitakami region on the Japanese main island as possible candidate. Project related evaluations on government level are ongoing.

The results - which are presented in the following - are taken from [4] and relate to the updated running scenario [3]. A preferred running scenario (fig. 1) foresees energies starting at $500 \mathrm{GeV}$, then going down to $350 \mathrm{GeV}$ before finally running at 250 $\mathrm{GeV}$. After a luminosity upgrade, the upgraded machine should run at $500 \mathrm{GeV}$ and $250 \mathrm{GeV}$, respectively. In total, it is planned to collect $2000 \mathrm{fb}^{-1}$ at $250 \mathrm{GeV}, 200 \mathrm{fb}^{-1}$ at $350 \mathrm{GeV}$, and $4000 \mathrm{fb}^{-1}$ at $500 \mathrm{GeV}$ during the full ILC programme. Nevertheless, the actual running scenario will depend on LHC physics results obtained by that time, as well as on the results of the first ILC runs.

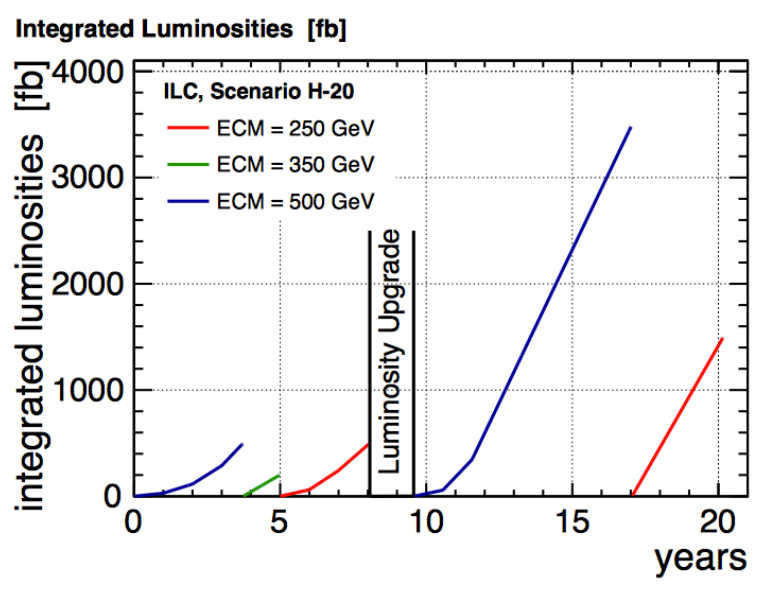

Figure 1: Preferred running scenario in the context of a 20 -year-long physics programme. Figure taken from [3].

\section{Higgs Production Processes and Observation}

Each collision energy gives access to different Higgs production processes, which in turn offer various precision measurements of Higgs properties. Single Higgs production processes and their cross sections are depicted in figure 2 as function of the centre-of-mass energy, where the dominant contributions arise from Higgs strahlung and WW fusion. At $250 \mathrm{GeV}$, the dominant production process is Higgs strahlung. The advantage of this production process is that it provides a model- 


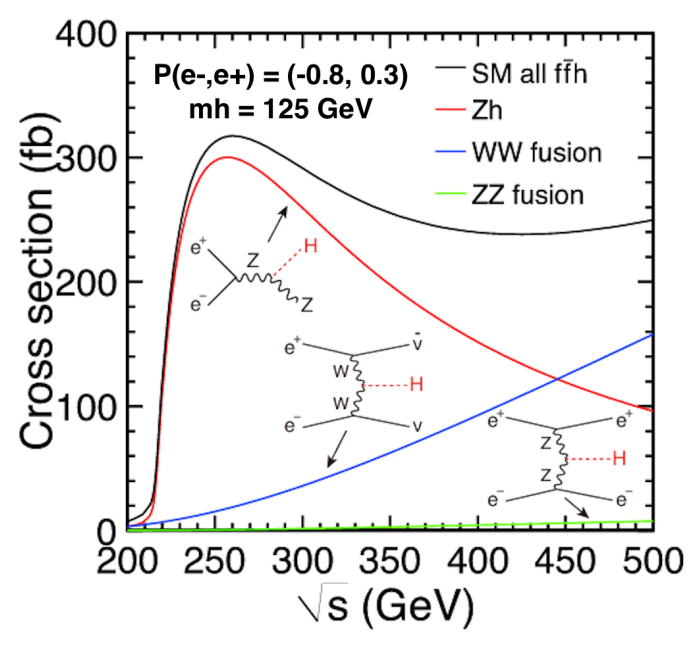

Figure 2: Single Higgs production processes at $e^{+} e^{-}$- colliders. Production cross sections as a function of the centre-of-mass energy $\sqrt{s}$ for a

$125 \mathrm{GeV}$ Higgs boson. Figure from [4].

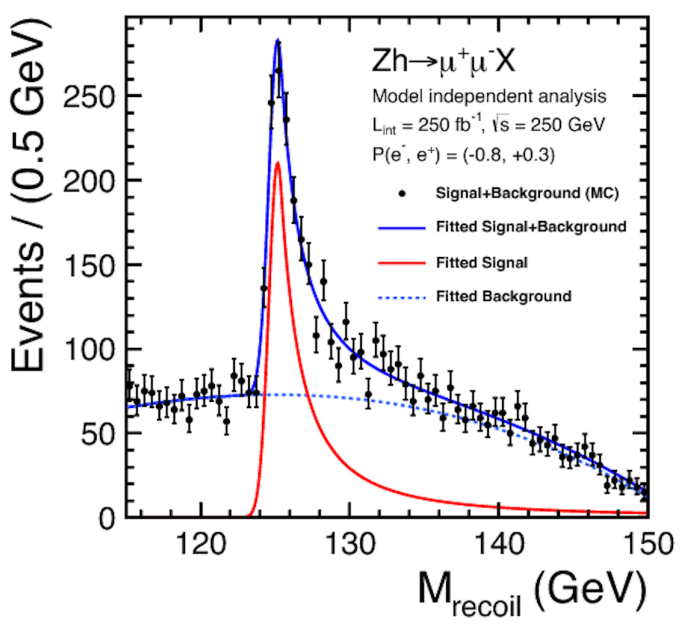

Figure 3: Higgs recoil mass measurement of $Z \rightarrow \mu^{+} \mu^{-}$. A precision on the Higgs mass of

$15 \mathrm{MeV}$ can be achieved after the full ILC programme. Figure taken from [10].

independent method to reconstruct Higgs events by the recoil mass $M_{\text {recoil }}^{2}=\left(p_{e^{+} e^{-}}-p_{Z}\right)^{2}$ from the leptonic decay products of the $Z$ boson $p_{Z}$, assuming $Z \rightarrow e^{+} e^{-} / \mu^{+} \mu^{-}$[10]. Here, $p_{e^{+} e^{-}}$is well-defined by the initial $e^{+} e^{-}$-system. The reconstructed recoil mass is depicted in figure 3 . This recoil mass technique is the key to the completely model-independent Higgs analysis at the ILC, since no Higgs reconstruction is required. Consequently, it offers the possibility to observe Higgs decays to invisible or exotic modes. Being sensitive to invisible Higgs decay channels, the technique provides a direct and model-independent measurement of the absolute cross section $\sigma_{Z H}$ to a precision at sub-\% level [11].

\subsection{Higgs Couplings}

Combining measurements of Higgs production cross sections and Higgs branching ratios gives the possibility to extract the total Higgs width $\Gamma_{\text {tot }}^{H}$ and Higgs couplings $g_{H X X}^{2}$ model-independently, where the production rate in a specific channel is given by

$$
\frac{N}{\mathscr{L}}=\sigma_{i} \cdot B R(H \rightarrow X X)=\sigma_{i} \cdot \frac{\Gamma(H \rightarrow X X)}{\Gamma_{t o t}^{H}} \propto \frac{g_{i}^{2} \cdot g_{H X X}^{2}}{\Gamma_{t o t}^{H}}
$$

In order to learn about the absolute sizes of all Higgs couplings, it is necessary to determine the total width $\Gamma_{\text {tot }}^{H}$ in a model-independent way. However, $\Gamma_{\text {tot }}^{H}$ is too small to be measured directly and thus an indirect approach has to be applied using WW-fusion events $v \bar{v} H$, assuming $H \rightarrow b \bar{b}$, at energies above $350 \mathrm{GeV}$ [12].

As shown in eq. 2.1, a measurement of the WW-fusion cross section $\sigma_{v \bar{v} H}$ can be transformed into a measurement of the coupling $g_{H W W}^{2}$ by dividing the production rate by $B R(H \rightarrow b \bar{b})$. One has to keep in mind that using the recoil mass technique, the measurement of $\sigma_{Z H}$ with Higgs-strahlung 


\begin{tabular}{l|c|c} 
& ILC500 & ILC500 LumiUP \\
\hline$\Gamma($ tot $)$ & $3.8 \%$ & $1.8 \%$ \\
$\mathrm{~g}(\mathrm{HZZ})$ & $0.58 \%$ & $0.31 \%$ \\
$\mathrm{~g}(\mathrm{HWW})$ & $0.81 \%$ & $0.42 \%$ \\
$\mathrm{~g}(\mathrm{Hbb})$ & $1.5 \%$ & $0.7 \%$ \\
$\mathrm{~g}(\mathrm{Hcc})$ & $2.7 \%$ & $1.2 \%$ \\
$\mathrm{~g}(\mathrm{Hgg})$ & $2.3 \%$ & $1.0 \%$ \\
$\mathrm{~g}(\mathrm{H} \tau \tau)$ & $1.9 \%$ & $0.9 \%$ \\
$\mathrm{~g}(\mathrm{H} \gamma \gamma)$ & $7.8 \%$ & $3.4 \%$ \\
$\mathrm{~g}(\mathrm{H} \mu \mu)$ & $20 \%$ & $9.2 \%$ \\
$\mathrm{~g}(\mathrm{Htt})$ & $18 \%$ & $6.3 \%$
\end{tabular}

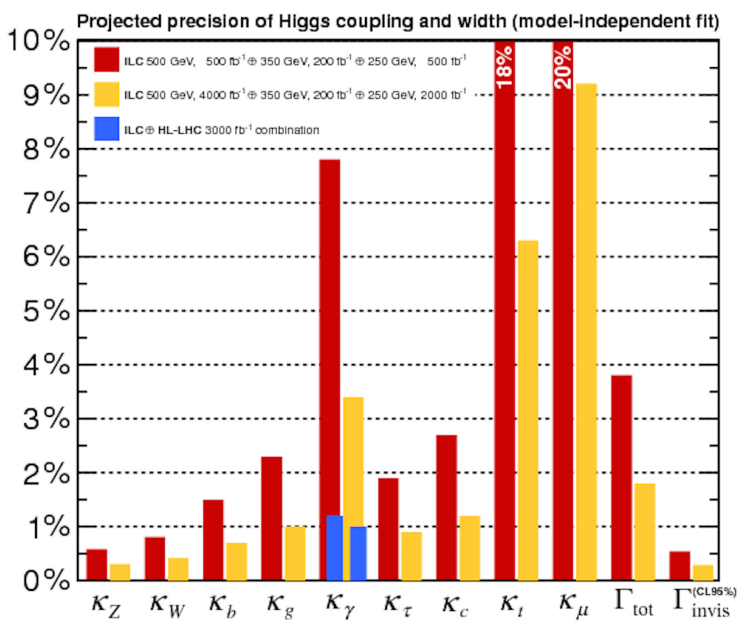

Figure 4: Projected precisions of SM Higgs measurements at the ILC for the updated runnings scenario [3].

The figure illustrates the obtained accuracies for the runnings stages before (red) and after (yellow) luminosity upgrade. The blue projections represent combined ILC and HL-LHC results of the coupling $H \rightarrow \gamma \gamma$. Figure and numbers taken from [4].

events yields the direct measurement of absolute branching ratios and the measurement of the coupling $g_{H Z Z}^{2}$ without any assumptions on $\Gamma_{\text {tot }}^{H}$. This implies an absolute measurement of the total Higgs width in a model-independent way. The former coupling $g_{H Z Z}^{2}$ can be measured to a precision of $0.31 \%$ after the full ILC programme [4, 11].

These measurements provide the determination of the partial decay widths $\Gamma(H \rightarrow W W)$ and $\Gamma(H \rightarrow Z Z)$, which then in turn offer a completely model-independent measurement of $\Gamma_{t o t}^{H}$ using the relation between total and partial width

$$
\Gamma_{t o t}^{H}=\Gamma(H \rightarrow X X) / B R(H \rightarrow X X) .
$$

An accuracy of $1.8 \%$ on $\Gamma_{t o t}^{H}$ can be achieved $[4,11]$. The coupling $g_{H W W}$ can be measured to a precision of $0.42 \%$ after the full ILC programme $[4,11]$.

At around $500 \mathrm{GeV}$, the production process $e^{+} e^{-} \rightarrow t \bar{t} H$ becomes accessible, allowing a model-independent measurement of the top Yukawa coupling. The coupling strength of the Higgs boson to fermions is proportional to the fermion masses in the SM. Measuring the top Yukawa coupling represents a test of this SM prediction due to the large top mass compared to other fermion masses. Additionally, new physics models predict deviations from the SM value, making this measurement an important test of new physics. At $500 \mathrm{GeV}$, the $t \bar{t} H$ production cross section is sharply rising and it is possible to reach a precision of $6.3 \%$ on the coupling after the full ILC programme. However, by increasing the centre-of-mass energy by $10 \%$ the precision on $g_{h t t}$ can be improved by a factor two to $3 \%$, assuming the same integrated luminosity [3].

The estimated uncertainties from ILC Higgs physics measurements, including Higgs decay modes to invisible and exotic modes, are summarised in figure 4. The precisions are obtained from a model-independent global fit, in which all couplings are taken as free parameters. Most of the couplings reach a precision at the sub-\% level, thus providing the potential to discover percent-level deviations with high significance. The accuracy on the top Yukawa coupling can be improved by a 

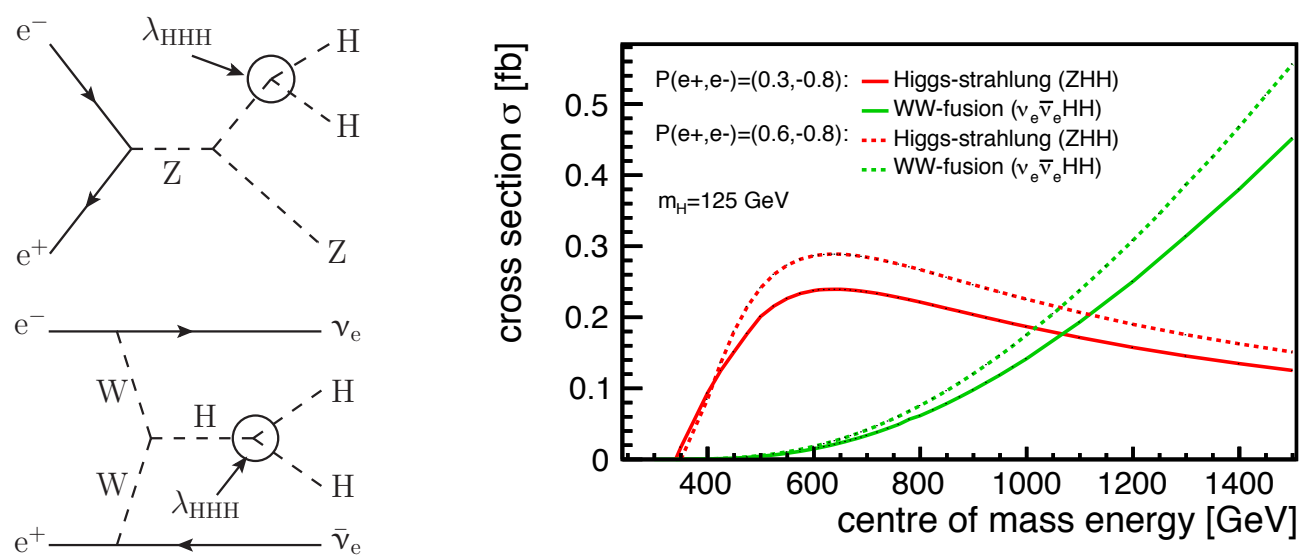

Figure 5: Left: Double Higgs production at $e^{+} e^{-}$-collider. Double Higgs strahlung (top) and WW fusion (bottom). Right: Production cross section vs. centre-of-mass energy. Figure taken from [13].

factor of two, when running the ILC at $550 \mathrm{GeV}$ rather than $500 \mathrm{GeV}$. In the case of the Higgs coupling to photons, a combination with LHC measurements can improve the coupling precision to $1.2 \%$ before the luminosity upgrade, and to $1.0 \%$ using the full ILC data set.

\subsection{Higgs Self-Coupling}

A precise measurement of the trilinear Higgs self-coupling is necessary to fully understand the mechanism of electroweak symmetry breaking and mass generation of fermions and gauge bosons. The Higgs self-coupling is important to establish the SM Higgs Mechanism experimentally and to reconstruct the potential of the Higgs field. At the ILC, the trilinear Higgs self-coupling can be measured directly in Higgs pair production. The production cross sections of double Higgs production processes are shown in figure 5, as well as the Feynman diagrams of double Higgs strahlung and WW fusion. Both production processes include irreducible background diagrams which degrade the coupling sensitivity. Combined with very small signal production cross sections and multi-jet final states, the measurement provides big challenges to the experiment.

At $500 \mathrm{GeV}$ information on the coupling can be extracted from a cross-section measurement of the double Higgs-strahlung process. Full detector simulations predict an achievable precision of $77 \%$ on the Higgs self-coupling at the ILC before the luminosity upgrade. After the full ILC programme, a precision of $27 \%$ is expected [3]. This corresponds to an evidence larger than $3 \sigma$ for the existence of the SM Higgs self-coupling.

An energy upgrade to $1 \mathrm{TeV}$ would offer complementary capabilities for the observation of double Higgs production and the Higgs self-coupling measurement, giving access to WW-fusion channel. Including WW-fusion events, the precision on the Higgs self-coupling measurement would improve to $10 \%$ or better [11]. There are several studies ongoing (overview in [13]), which investigate and implement several areas for improvement in the measurement.

Moreover, the trilinear Higgs self-coupling is one of the most important discriminative quantities to reveal new physics models. In several extended Higgs theories the selfcoupling can deviate by more than a few percent from SM predictions. Models of electroweak baryogengesis in the framework of Two Higgs Doublet Models (2HDM) [14] ex- 
pect minimal deviations of $20 \%$ from SM predictions, for example, as it is illustrated in figure 6. In this example, the Higgs self-coupling could even be twice as high as in the SM.

Since both double Higgs production channels, WW fusion and Higgs strahlung, are complementary in their sensitivity to new physics due to the sign of the interference term, double Higgs strahlung and WW fusion behave very different with respect to changes in the Higgs self-coupling. A Higgs self-coupling value smaller than the SM prediction can be observed with WW fusion, whereas double Higgs-strahlung events provide measurements of new physics scenarios with a trilinear Higgs coupling larger than the predicted SM value.

In scenarios with electroweak baryogengesis, as depicted in figure 6, the expected deviation in the Higgs self-coupling from the SM value results in a larger cross

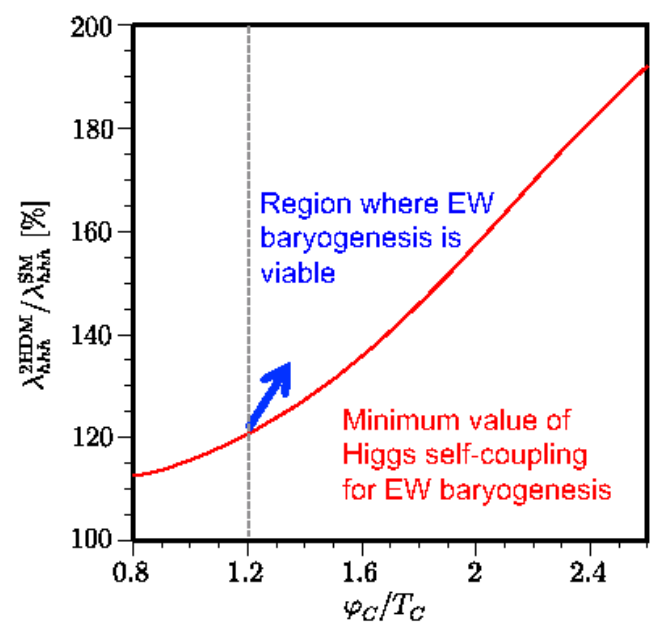

Figure 6: Possible values of the Higgs self-coupling normalised to the SM value, in the framework of Two Higgs Doublet Models with electroweak baryogenesis. A minimum deviation from the SM is in the order of 20\%. Figure from [14]. section for double Higgs strahlung and in a smaller cross section for the WW-fusion process $[4,13]$. Thus, scenarios with electroweak baryogengesis are possible to be observed at the ILC at $500 \mathrm{GeV}$ with double Higgs strahlung to very high precision [13].

\section{Summary}

The ILC provides direct precision measurements of Higgs boson properties without any modeldependence, which is essential for the reconstruction of the electroweak symmetry breaking sector and to reveal new physics. Very high precision is required in order to reveal deviations from the SM predictions and get insight to new physics. Recently, different operating scenarios were studied, providing a staged running programme with different centre-of-mass energies, each of which gives access to different Higgs production processes. The various Higgs production channels provide model-independent measurements of all Higgs couplings and the total Higgs width to very high precision. Additionally, the measurement of double Higgs production and thus the Higgs selfcoupling is possible at the ILC. Full detector simulation studies show that it is possible to observe double Higgs production and obtain scientific evidence of the existence of the SM Higgs selfcoupling. With regard to new physics models predicting deviations from the SM self-coupling value, the ILC provides a great discovery potential already at $500 \mathrm{GeV}$ centre-of-mass energy.

The ILC moved to the engineering stage, with site specific evaluations in Japan. Political reviews of the ILC project on government level are taking place, the term of which are set to be earliest in 2016, depending on the LHC physics results by that time. 


\section{References}

[1] G. Aad et al. [ATLAS Collaboration], Observation of a new particle in the search for the Standard Model Higgs boson with the ATLAS detector at the LHC, Phys. Lett. B 716 (2012) 1, 1207.7214.

[2] S. Chatrchyan et al. [CMS Collaboration], Observation of a new boson at a mass of $125 \mathrm{GeV}$ with the CMS experiment at the LHC, Phys. Lett. B 716 (2012) 30, 1207.7235.

[3] T. Barklow, J. Brau, K. Fujii, J. Gao, J. List, N. Walker and K. Yokoya, ILC Operating Scenarios, 1506.07830.

[4] K. Fujii et al., Physics Case for the International Linear Collider, 1506.05992.

[5] H. Baer, T. Barklow, K. Fujii, Y. Gao, A. Hoang, S. Kanemura, J. List and H. E. Logan et al., The International Linear Collider Technical Design Report - Volume 2: Physics, 1306.6352.

[6] T. Behnke, J. E. Brau, B. Foster, J. Fuster, M. Harrison, J. M. Paterson, M. Peskin and M. Stanitzki et al., The International Linear Collider Technical Design Report - Volume 1: Executive Summary, 1306.6327.

[7] C. Adolphsen, M. Barone, B. Barish, K. Buesser, P. Burrows, J. Carwardine, J. Clark and H. M. Durand et al., The International Linear Collider Technical Design Report - Volume 3.I: Accelerator \& in the Technical Design Phase, 1306.6353.

[8] C. Adolphsen, M. Barone, B. Barish, K. Buesser, P. Burrows, J. Carwardine, J. Clark and H. M. Durand et al., The International Linear Collider Technical Design Report - Volume 3.II: Accelerator Baseline Design, 1306.6328.

[9] T. Behnke, J. E. Brau, P. N. Burrows, J. Fuster, M. Peskin, M. Stanitzki, Y. Sugimoto and S. Yamada et al., The International Linear Collider Technical Design Report - Volume 4: Detectors, 1306.6329.

[10] H. Li et al. [ILD Design Study Group Collaboration], HZ Recoil Mass and Cross Section Analysis in ILD, 1202.1439.

[11] D. M. Asner et al., ILC Higgs White Paper,1310.0763.

[12] C. Dürig, K. Fujii, J. List and J. Tian, Model Independent Determination of HWW coupling and Higgs total width at ILC, 1403.7734.

[13] C. Dürig, Update on the Higgs self-coupling analyses for ILD, presentation at the Asian Linear Collider Workshop 2015.

[14] S. Kanemura, E. Senaha, T. Shindou and T. Yamada, Electroweak phase transition and Higgs boson couplings in the model based on supersymmetric strong dynamics, JHEP 1305 (2013) 066, 1211.5883. 